\title{
FATORES CRÍTICOS DE SUCESSO (FCS) NO FINAL DO CICLO DE VIDA DE PRODUTOS
}

\author{
Samuel Borges Barbosa, MSc. (UFSC); \\ Marcelo Gitirana Gomes Ferreira, Dr. (UFSC)
}

\begin{abstract}
PALAVRAS-CHAVE
sustentabilidade, final do ciclo de vida do produto, fatores críticos de sucesso.

KEYWORDS

sustainability, product end-of-life, success critical factors.
\end{abstract}

\section{RESUMO}

Desafios relacionados à degradação do meio ambiente têm gerado discussões sobre a destinação correta dos produtos após o uso. Neste sentido um grande número de processos tem sido aplicado no setor produtivo industrial, visando criar soluções para a correta destinação dos produtos e seus materiais no final do ciclo de vida. Porém, além da aplicação destes processos é necessário desenvolver métodos de gestão que busquem aprimorá-los. A pesquisa apresentada tem como objetivo identificar Fatores Críticos de Sucesso (FCS) envolvidos nos processos do final do ciclo de vida do produto, no setor de bens duráveis. Estes processos foram identificados a partir de um levantamento bibliométrico. Foi elaborado um plano para a coleta dos referenciais teóricos, sendo utilizados portais de pesquisas disponíveis para universidades públicas brasileiras. Após a identificação dos FCS foi aplicada uma validação destes perante especialistas do setor de desenvolvimento de produtos. A realização deste trabalho proporcionou uma reflexão sobre os processos envolvidos no final do ciclo de vida do produto, além de caracterizar-se como um ponto de partida para futuras pesquisas que visem desenvolver métodos de gestão que apoiem a meIhoria destes processos.

\footnotetext{
ABSTRACT

Challenges related to environmental degradation have generated discussions about the proper disposal of products after use. In this sense a large number of processes have been applied in the industrial sector, to create solutions for the proper disposal of products and materials at the end of their life cycle. However, besides the application of these processes it is necessary to develop management methods that seek to improve these processes. The research aims to identify Critical Success Factors (CSF) involved in the end of life cycle processes, in the durable goods sector. These processes were identified from a bibliometric survey. A plan was elaborated to identify theoretical researches in the field of product end of life. After identifying the FCS a validation was applied, by a consult of experts in the field of product development. This research provided a reflection on the processes involved at the product end of life cycle, and can be characterized as a starting point for the development of management tools to improve these processes.
} 


\section{INTRODUÇÃO}

Preocupações ambientais referentes à degradação do meio ambiente, causada pela produção industrial, têm gerado discussões em vários setores da sociedade. $\mathrm{O}$ aumento do consumo, o qual funciona como um propulsor na produção de bens, gera o aumento da oferta de produtos no mercado. Dessa maneira, órgãos do governo, empresas, organizações não governamentais e outras instituições tem demonstrado empenho constante em prol da desaceleração da produção e da criação de soluções que diminuam as interferências geradas no meio ambiente pelo desenvolvimento industrial (KOTLER, 1996).

Nesse contexto surge a visão da sustentabilidade e do desenvolvimento sustentável, que propõem o crescimento e desenvolvimento econômico atual sem prejuízos para as gerações futuras. Esta ideia parte do princípio de que mesmo consumindo recursos naturais hoje é necessário que sejam mantidas condições para que as futuras gerações possam também satisfazer suas necessidades (MANZINI e VEZZOLI, 2002).

Alguns métodos que visam reduzir os impactos ambientais causados pela produção de bens surgiram nas últimas décadas, sendo inicialmente propostos em estudos e posteriormente aplicados no âmbito industrial. Especialmente na área de bens duráveis, vários processos têm sido aplicados em diversas empresas, como remanufatura, reciclagem e o reuso de produtos. Estes processos interferem diretamente na fase final do ciclo de vida dos produtos (product end of life) (BELLMANN e KHARE, 2000; HOEKSTRA, 2015).

Neste contexto, este estudo apresenta uma revisão bibliográfica com objetivo de identificar um conjunto de fatores críticos de sucesso (FCS) relacionados ao final do ciclo de vida de produtos do setor de bens duráveis. Para isto é feito primeiramente um levantamento estruturado de publicações, buscando encontrar trabalhos que apresentem referências quanto aos processos envolvidos no final do ciclo de vida do produto. Em seqüência, com base nas publicações encontradas, é feita uma seleção de trabalhos e uma avaliação quanto ao conteúdo destes, buscando identificar as referências quanto aos processos. Por final é feita a identificação dos FCS e a validação destes perante especialistas do setor.

\section{METODOLOGIA}

A pesquisa bibliográfica (GIL, 2002) foi utilizada nesta pesquisa como forma de fundamentar os conteúdos teóricos envolvidos na pesquisa. A pesquisa bibliométrica também foi aplicada, servindo como base para a identificação das publicações. O termo bibliometria refere-se às análises quantitativas dos padrões que aparecem nas publicações e o uso de documentos (DIODATO, 1994). Por meio da análise bibliométrica é possível identificar a freqüência na quais certos trabalhos aparecem, mostrando assim a sua influência na área específica (BORGMAN \& FURNER, 2002). Com o desenvolvimento da tecnologia da informação, ocorrida nos últimos anos, as pesquisas bibliográficas podem ser feitas por meio de bases de dados digitais, as quais são formadas por conjuntos de publicações como livros, artigos, resumos de artigos entre outros documentos (VANTI, 2002).

Todas as publicações levantadas neste trabalho estão disponíveis nas bases de dados presentes no Portal de Periódicos da CAPES (Coordenação de Aperfeiçoamento de Pessoal de Nível Superior) e na base Science Direct. Foram analisados apenas os artigos disponíveis para as universidades federais brasileiras. As bases de dados selecionadas para a coleta foram: Science Direct, EBSCO, Emerald, CSA, Wilson, Google Scholar, IEEE Xplore, Scielo, SpringerLink e Web of Science.

\section{FINAL DO CICLO DE VIDA DO PRODUTO}

Atualmente algumas iniciativas têm sido tomadas no setor produtivo buscando reduzir os impactos ambientais gerados pelo descarte de produtos. Para exemplificar estas iniciativas 
podemos citar a diretiva que impõe responsabilidade ao fabricante para o lixo proveniente de equipamentos elétricos e eletrônicos, sendo esta aplicada atualmente na Europa. Esta diretiva aplica regras para o tratamento e a correta destinação de produtos eletrônicos e elétricos após o seu uso (RAHIMIFARD, ABU BAKAR e WILLIAMS, 2009).

Outros produtos, diferentemente de equipamentos elétricos e eletrônicos, também têm tido um tratamento especial após o seu uso, visando o reaproveitamento de materiais e energia. Um desses casos é o de pneus em Portugal, apresentado por Ferrão, Ribeiro e Silva (2008). Neste caso específico, processos como coleta de produtos, retalhamento, trituração, moagem, separação magnética, separação por moagem, incineração e aterramento formam a fase final do ciclo de vida do produto, onde uma parte dos materiais é reaproveitada e outra é descartada. Outros produtos que também têm tido, no final do seu ciclo de vida, um tratamento voltado à recuperação de materiais e correta destinação de resíduos são: veículos (BELLMAN e KHARE, 2000; FORSLIND, 2005; SAKAI, NOMA e KIDA, 2007), telefones celulares (OSIBANJO e NNOROM, 2008), televisores (ANDREOLA et al., 2007).

Porém, apesar da vasta gama de trabalhos encontrados que buscam discutir e estruturar os processos no final do ciclo de vida dos produtos, poucos propõem maneiras de gerenciá- los de maneira que possa haver uma contínua melhoria dos processos. Wright, Rahimifard e Clegg (2009) assumem em seu trabalho, onde analisam os impactos da legislação ambiental na fabricação de células de combustível, que é necessário desenvolver indicadores para a classificação de resíduos perigosos e para o controle de como os resíduos são tratados. Os mesmos autores também citam o Gerenciamento Ambiental Responsável de produtos como de grande importância para vários tipos de produtos, como componentes elétricos e eletrônicos, baterias e componentes automotivos.

Dessa maneira é possível indicar, pela análise da literatura apresentada, a necessidade de desenvolvimento de ferramentas que sirvam de suporte para o gerenciamento do final do ciclo de vida dos produtos. Porém para isso é necessário entender quais processos estão atualmente envolvidos na recuperação destes produtos e como deve ser estruturado o sistema formado por eles. Para isso será apresentado neste artigo uma análise de publicações que tenham sido desenvolvidos com o intuito de mapear os processos envolvidos no final do ciclo de vida do produto. Assim será possível estruturar um modelo genérico dos processos que possa ser usado como base para a construção de um sistema de gerenciamento do final do ciclo de vida do produto, focando no presente trabalho no setor de bens duráveis.

\section{CONSULTA BIBLIOGRÁFICA}

A consulta bibliográfica aplicada teve como objetivo a identificação de publicações relevantes dentro do seguinte tema: gerenciamento do final do ciclo de vida de produtos. A pesquisa foi feita utilizando duas ferramentas de busca: o portal de periódicos CAPES e o portal Science Direct. As bases de dados consultadas foram as seguintes: Science Direct, EBSCO, Emerald, CSA, Wilson, Google Scholar, IEEE Xplore, Scielo, SpringerLink e Web of Science.

Para realizar a busca foi necessário definir o termo (string) que seria utilizado. Primeiramente é necessário estabelece o ambiente da pesquisa, o qual foi definido como o final do ciclo de vida do produto. Sendo o gerenciamento dos processos envolvidos no final do ciclo de vida o objetivo deste trabalho foi possível definir a expressão "gerenciamento do final do ciclo de vida do produto" ("end of life product management"). Assim foi estabelecido este termo como o direcionador da pesquisa, tendo sido utilizada a sua tradução em inglês, pois as bases consultadas possuem periódicos internacionais escritos nesta língua.

A busca realizada no portal de periódicos CAPES obteve 155 registros. Esses registros representam 155 publicações provenientes das bases, sendo estas publicações em revistas internacionais e publicações em eventos interna- 
cionais. Já a busca realizada no portal do Science Direct obteve 60 registros. Esses resultados representam 60 publicações provenientes de revistas internacionais.

Após a eliminação dos resultados duplicados foi obtido um total de 128 publicações.

Porém, nem todas as publicações obtidas apresentaram utilidade para o propósito do trabalho, que é encontrar referências de processos existentes no final do ciclo de vida do produto. Assim, foi necessário criar um sistema de análise para avaliar se a publicação possuía as informações necessárias para ser analisada. $O$ sistema de análise utilizado seguiu dois passos: (1) as publicações teriam seus títulos, resumos e palavras-chave analisados por meio de leitura; (2) se estes mostrassem favoráveis ao assunto buscado seria feito uma análise do conteúdo da publicação, com o intuito de achar gráficos e tabelas que apontassem para a possível presença de informações na publicação sobre processos envolvidos no final do ciclo de vida do produto.

Feitas estas análises, foi encontrado um de importância para o trabalho. Estas publicações teriam todo seu conteúdo analisado por meio de leitura.

\section{IDENTIFICAÇÃO DOS PROCESSOS ENVOL- VIDOS NO FINAL DO CICLO DE VIDA}

Após a aplicação da busca foram identificados nas publicações os processos envolvidos no final do ciclo de vida dos produtos. Junto com a identificação dos processos foram identificados também os produtos relacionados. As publicações obtidas se referem a uma grande variedade de produtos diferentes, como eletrônicos, pneus, computadores, automóveis, lâmpadas, eletrodomésticos, dentre outros, sendo na sua maioria classificados como bens duráveis. Com relação aos processos, somente 14 publicações apresentam uma linha de processos que deve ser utilizado na fase de final do ciclo de vida do produto. A lista com os produtos e processos identificados está presente no quadro 1.

Quadro 1 - Produtos e Processos Relacionados nas Publicações.

\begin{tabular}{|c|c|c|c|c|c|c|c|c|c|c|c|c|c|c|c|c|c|c|c|c|c|c|c|c|c|c|c|c|c|c|}
\hline & & & Pro & dut & & & & & & & & & & & & & & roc & cess & & & & & & & & & & & \\
\hline Referências & 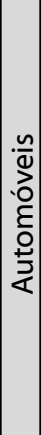 & 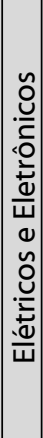 & 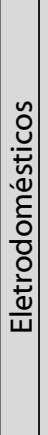 & 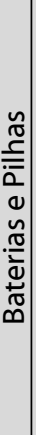 & 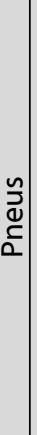 & $\begin{array}{l}\frac{n}{\pi} \\
\frac{0}{2} \\
\sum\end{array}$ & 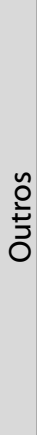 & 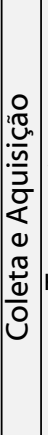 & 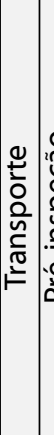 & 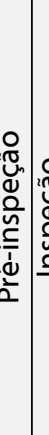 & 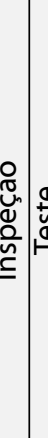 & $=$ & 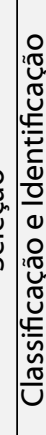 & 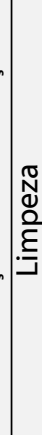 & 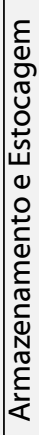 & 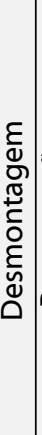 & 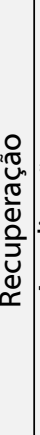 & 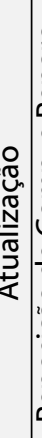 & 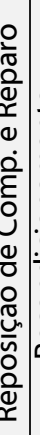 & 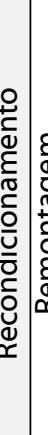 & 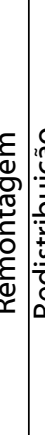 & & 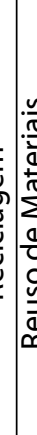 & 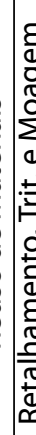 & 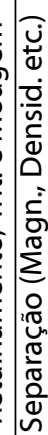 & 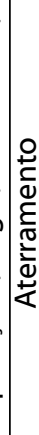 & 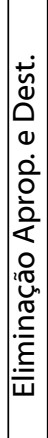 & 0 & 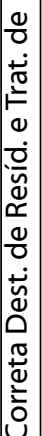 & مِّ \\
\hline Goggin e Browne (1998) & & $\mathrm{X}$ & & & & & & $\mathrm{x}$ & & & & & & & & $\bar{x}$ & & $\mathrm{X}$ & & $\bar{x}$ & & & $\overline{2}$ & & $\mathrm{X}$ & & $\mathrm{X}$ & & & \\
\hline Grenchus et al., (1998) & & & & & & & $\mathrm{X}$ & & & & & & & & & & & & & & & & & & & & & & & \\
\hline Low, Williams e Dixon (1998) & & & & & & & $\mathrm{X}$ & & & & & & & & & & & & & & & & & & & & & & & \\
\hline Gungor e Gupta (1999) & & & & & & & $\mathrm{X}$ & & & & & & & & & & & & & & & & & & & & & & & \\
\hline Yu et al. (2000) & & $\mathrm{X}$ & & & & & & & & & & & & & & & & & & & & & & & & & & & & \\
\hline Bellmann e Khare (2000) & $\mathrm{X}$ & & & & & & & & & & & & & & & & & & & & & & & & & & & & & \\
\hline Fthenakis (2000) & & & & & & & $\mathrm{X}$ & & & & & & & & & $\mathrm{X}$ & $\mathrm{X}$ & & & & & & & $x$ & & & & & & $\mathrm{x}$ \\
\hline Caudill et al. (2000) & $\mathrm{X}$ & & & & & & & & & & & & & & & & & & & & & & & & & & & & & \\
\hline Sutherland, Gunter e Weinmann (2001) & & & & & & & $\mathrm{X}$ & & & & & & & & & & & & & & & & & & & & & & & \\
\hline
\end{tabular}




\begin{tabular}{|c|c|c|c|c|c|c|c|c|c|c|c|c|c|c|c|c|c|c|c|c|c|c|c|c|c|c|c|c|c|}
\hline Rose, Ishii e Stevels (2001) & & & & & $\mathrm{X}$ & & & & & & & & & & & & & & & & & & & & & & & & \\
\hline Mangun e Thurston (2002) & & $\mathrm{X}$ & & & & & & & & & & & & & & & & & & & & & & & & & & & \\
\hline White et al. (2003) & & $\mathrm{X}$ & & & & & $\mathrm{X}$ & $\mathrm{X}$ & & & & $\mathrm{X}$ & & & $\mathrm{X}$ & & & & & & & & & & & & & & \\
\hline Dickinson e Caudill (2003) & & & & & & $\mathrm{x}$ & & & & & & & & & & & & & & & & & & & & & & & \\
\hline Qian e Zhang (2003) & & & & & & $\mathrm{x}$ & & & & & $\mathrm{X}$ & & $\mathrm{X}$ & & & $\mathrm{X}$ & & & & & & & & $\mathrm{X}$ & $\mathrm{x}$ & \begin{tabular}{l|l}
$x$ \\
\end{tabular} & $\mathrm{X}$ & & $\bar{x}$ \\
\hline Masanet e Horvath (2004) & & $\mathrm{X}$ & & & & & & & & & & & & & & & & & & & & & & & & & & & \\
\hline Caudill e Dickinson (2004) & & $\mathrm{x}$ & & & & & & & & & & & & & & & & & & & & & & & & & & & \\
\hline Laszewski e Carey (2004) & & $\mathrm{X}$ & & & & & & & & & & & & & & & & & & & & & & & & & & & \\
\hline Forslind (2005) & $\mathrm{X}$ & & & & & & & & & & & & & & & & & & & & & & & & & & & & \\
\hline Peralta e Fontanos (2006) & & $x$ & & & & & $\mathrm{x}$ & & & & & & & & $\mathrm{X}$ & & $\mathrm{X}$ & & & & & $\mathrm{X}$ & $\bar{X}$ & & & $\mathrm{X}$ & & $x$ & \\
\hline Nakamura e Kondo (2006) & & & $\mathrm{X}$ & & & & & & & & & & & & & & & & & & & & & & & & & & \\
\hline Ruhrberg (2006) & & & & & $\mathrm{X}$ & & & & & & & & & & & & & & & & & & & & & & & & \\
\hline $\begin{array}{c}\text { Zwolinski, Lopez-Ontiveros } \\
\text { e Brissaud (2006) }\end{array}$ & & & & & & $\mathrm{X}$ & & & & & & & & & & & & & & & & & & & & & & & \\
\hline Pagell, Wu e Murthy (2007) & & & & & & $x$ & & & & & & & & & & & & & & & & & & & & & & & \\
\hline Abu Bakar e Rahimifard (2007) & & $\mathrm{X}$ & & & & & & & & & & & & & $\mathrm{X}$ & & & $\mathrm{X}$ & $\mathrm{X}$ & & & $\mathrm{X}$ & \begin{tabular}{l|l}
$X$ & $\gamma$ \\
\end{tabular} & $\mathrm{X}$ & & & $\mathrm{X}$ & $x$ & \\
\hline Duval e Maclean (2007) & $x$ & & & & & & & & & & & & $\mathrm{X}$ & & $\mathrm{X}$ & & & & & & & & & $\bar{x}$ & & & & & \\
\hline Parsons (2007) & & & & $\mathrm{X}$ & & & & & & & & & & & & & & & & & & & & & & & & & \\
\hline Um, Yoon e Suh (2008) & & & & & & $\mathrm{x}$ & & & $x$ & $\mathrm{X}$ & & & & & & & & $\mathrm{X}$ & $\mathrm{x}$ & & & $\mathrm{X}$ & & & & & & & \\
\hline Ferrão, Ribeiro e Silva (2008) & & & & & $\mathrm{X}$ & & $\mathrm{x}$ & & & & & & & & & & & & & & & & & 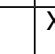 & \begin{tabular}{l|l}
$\mathrm{x}$ & $\mathrm{x}$ \\
\end{tabular} & & $\mathrm{X}$ & & \\
\hline Kumar e Putnam (2008) & $\mathrm{x}$ & $\mathrm{X}$ & $\mathrm{X}$ & & & & & & & & & & & & & & & & & & & & & & & & & & \\
\hline Kahhat et al. (2008) & & $\mathrm{X}$ & & & & & & & & & & & & & & & & & & & & & & & & & & & \\
\hline Johansson e Brodin (2008) & & $\mathrm{x}$ & & & & & & & & & & & & & & & & & & & & & & & & & & & \\
\hline Gehin, Zwolinski e Brissaud (2008) & & $\mathrm{X}$ & & & & & & & & & & & & & & & & & & & & & & & & & & & \\
\hline Cagno, Magalini e Trucco (2008) & & & $\mathrm{X}$ & & & & & & & & & & & & & & & & & & & & & & & & & & \\
\hline Duflou et al. (2008) & & $x$ & & & & & & & & $\mathrm{x}$ & & & $\mathrm{X}$ & $\mathrm{x}$ & & $\mathrm{x}$ & & & & & & & & $\mathrm{X}$ & & & & & \\
\hline Srogi (2008) & $\mathrm{X}$ & & & & & & & & & & & & & & & & & & & & & & & & & & & & \\
\hline Bettley e Burnley (2008) & & & & & & $\mathrm{X}$ & $\mathrm{x}$ & & & $\mathrm{X}$ & $\mathrm{X}$ & & & & & & $\mathrm{X}$ & & & & $x$ & & & $\gamma$ & $\mathrm{X}$ & & & & \\
\hline Wright, Rahimifard e Clegg (2009) & & & & & & $x$ & & & & & & & & & & & & & & & & & & & & & & & \\
\hline Amelia et al. (2009) & $\mathrm{X}$ & & & & & & & & & & & & & & & & & & & & & & & & & & & & \\
\hline Grujicic (2009) & $x$ & & & & & & & & & & & & & & & & & & & & & & & & & & & & \\
\hline lakovou et al. (2009) & & $\mathrm{X}$ & & & & & & & & & & & & & & $x$ & & & & & & & & & & & & & \\
\hline Xanthopoulos e lakovou (2009) & & & $\mathrm{X}$ & & & & & & & & & & & & & & & & & & & & & & & & & & \\
\hline Kuo (2010a) & & $x$ & & & & & & & & & & & & & & & & & & & & & & & & & & & \\
\hline Kuo (2010b) & & $\mathrm{X}$ & & & & & & & & & & & $\mathrm{X}$ & & & $x$ & & & & & & \begin{tabular}{l|l}
$x$ & $x$ \\
\end{tabular} & $\bar{X}$ & $\bar{X}$ & & $\mathrm{X}$ & & $x$ & \\
\hline Pigosso et al. (2010) & & & & & & $\mathrm{X}$ & & & & $\mathrm{X}$ & $\mathrm{X}$ & & & $\mathrm{X}$ & & $x$ & & $\mathrm{X}$ & $x$ & $\mathrm{X}$ & & & & & & & & & \\
\hline
\end{tabular}

Fonte: Autores.

Com a identificação dos processos foi feita uma categorização, de forma a criar um quadro genérico dos processos envolvidos no final do ciclo de vida, visando o setor de bens duráveis. Esta categorização é apresentada no quadro 2 . Os processos foram divididos em três etapas: etapa inicial, remanufatura e reciclagem.

\section{IDENTIFICAÇÃO DOS FATORES CRÍTICOS DE SUCESSO (FCS)}

Após a identificação dos processos relacionados à fase de final do ciclo de vida dos produtos e da estruturação modelo genérico dos processos foi necessários identificar os FCS. Para esta identificação foi utilizado o mesmo método apresentado para os processos. Foi feita a leitura das 46 publicações 
Quadro 2 - Processos Relacionados nas Publicações e Número de Citações.

\begin{tabular}{|c|c|c|}
\hline $\begin{array}{c}\text { Etapa do Final do } \\
\text { Ciclo de Vida do } \\
\text { Produto }\end{array}$ & Processo & Número de Citações \\
\hline \multirow{10}{*}{ Etapa 1 - Etapa Inicial } & Coleta e Aquisição & 5 \\
\hline & Transporte & 1 \\
\hline & Pré-Inspeção & 1 \\
\hline & Inspeção & 4 \\
\hline & Teste & 3 \\
\hline & Seleccão & 1 \\
\hline & Classificação e ldentificação & 4 \\
\hline & Limpeza & 2 \\
\hline & Armazenamento e Estocagem & 4 \\
\hline & Desmontagem & 7 \\
\hline \multirow{6}{*}{ Etapa 2 - Remanufatura } & Recuperação & 3 \\
\hline & Atualização & 4 \\
\hline & Reposição de Componentes e Reparo & 3 \\
\hline & Recondicionamento & 1 \\
\hline & Remontagem & 1 \\
\hline & Redistribuição & 1 \\
\hline \multirow{9}{*}{ Etapa 3 - Reciclagem } & Reciclagem & 4 \\
\hline & Reuso de Materiais & 4 \\
\hline & Retalhamento. Trituracão e Moagem & 5 \\
\hline & Separação (Magnética, Densidade etc.) & 4 \\
\hline & Aterramento & 2 \\
\hline & Eliminação Apropriada e Destruição & 4 \\
\hline & Incineração & 3 \\
\hline & Correta Dest de Resíd.e Trat de Substâncias Perigosas & 3 \\
\hline & Derretimento & 2 \\
\hline
\end{tabular}

Fonte: Autores.

identificadas na busca (quadro 3).

Foram encontrados nas publicações um conjunto de 40 FCS. Os FCS identificados podem ser divididos em cinco dimensões: (1) Identificação; (2) Eficiência na Recuperação; (3) Informações; (4) Incentivos e Regulamentação; (5) Resíduos Perigosos; (6) Outros. Este grupamento dos FCS foi possível por meio da aglomeração de características similares entre estes. Porém, de todos os FCS identificados alguns são citados um maior núme- ro de vezes nas publicações. Para a caracterização dos FCS com maior importância foi selecionado um número base de citações: 4 (maior ou igual a quatro citações). Assim, caracterizaram-se os FCS de maior importância como os que são citados quatro ou mais vezes. Isto foi feito para que seja possível a concentração do trabalho nos fatores que são chave para o gerenciamento do final do ciclo de vida do produto perante os aspectos relacionados à sustentabilidade ambiental.

Quadro 3 - Processos Relacionados nas Publicações e Número de Citações.

\begin{tabular}{|c|c|c|c|}
\hline Dimensão & $\begin{array}{c}\text { Fator Crítico de } \\
\text { Sucesso }\end{array}$ & Referência & Produtos \\
\hline \multirow{3}{*}{ Identificação } & $\begin{array}{l}\text { Identificação dos } \\
\text { Produtos (Marca, } \\
\text { Modelo) }\end{array}$ & $\begin{array}{l}\text { White et al. (2003); Goggin e } \\
\text { Browne, (1998); Johansson e } \\
\text { Brodin (2008); Qian e Zhang }\end{array}$ & $\begin{array}{l}\text { Computadores; Eletrônicos; Elétricos } \\
\text { e Eletrônicos; Eletromecânicos }\end{array}$ \\
\hline & $\begin{array}{l}\text { Identificação dos } \\
\text { Componentes }\end{array}$ & $\begin{array}{l}\text { lakovou et al. (2009); lakovou } \\
\text { et al., (2009); Goggin e Browne } \\
\text { (1998); Pigosso et al. (2010); } \\
\text { Duval e Maclean (2007); } \\
\text { Grenchus et al (1998) }\end{array}$ & $\begin{array}{l}\text { Eletrônicos; Eletrônicos; Geral; } \\
\text { Plásticos Automotivos (Geral); Geral }\end{array}$ \\
\hline & $\begin{array}{l}\text { Identificação dos } \\
\text { Materiais }\end{array}$ & $\begin{array}{c}\text { Goggin e Browne (1998); } \\
\text { Pigosso et al., (2010); } \\
\text { Johansson e Brodin (2008); } \\
\text { Grenchus et al (1998) }\end{array}$ & $\begin{array}{l}\text { Eletrônicos; Geral; Elétricos e } \\
\text { Eletrônicos; Geral }\end{array}$ \\
\hline
\end{tabular}




\begin{tabular}{|c|c|c|c|}
\hline \multirow{4}{*}{$\begin{array}{l}\text { Eficiência na } \\
\text { Recuperação }\end{array}$} & $\begin{array}{l}\text { Peso de Material } \\
\text { Recuperado por } \\
\text { Produto }\end{array}$ & $\begin{array}{c}\text { lakovou et al. (2009); } \\
\text { Wright, Rahimifard e Clegg } \\
\text { (2009); Pigosso et al. (2010); } \\
\text { Xanthopoulos e lakovou } \\
\text { (2009); Masanet e Horvath } \\
(2004)\end{array}$ & $\begin{array}{c}\text { Eletrônicos; Célula de Combustível; } \\
\text { Geral; Geral; Geral; Aquecedores } \\
\text { Elétricos; Plástico Proveniente de } \\
\text { Eletrônicos (Material) }\end{array}$ \\
\hline & $\begin{array}{l}\text { Quantidade de } \\
\text { Material Recuperável } \\
\text { por Produto }\end{array}$ & $\begin{array}{l}\text { Yu et al. (2000); Ruhrberg } \\
\text { (2006); Ruhrberg (2006); } \\
\text { Wright, Rahimifard e Clegg } \\
\text { (2009); White et al. (2003); } \\
\text { Pigosso et al. (2010); Pigosso } \\
\text { et al. (2010); Masanet e } \\
\text { Horvath (2004) }\end{array}$ & $\begin{array}{l}\text { Eletrônicos; Cobre (Material); Cobre } \\
\text { (Material); Células de Combustível; } \\
\text { Computadores; Geral; Geral; Plástico } \\
\text { Proveniente de Eletrônicos (Material) }\end{array}$ \\
\hline & $\begin{array}{l}\text { Tempo Necessário } \\
\text { para Recuperação do } \\
\text { Produto }\end{array}$ & $\begin{array}{l}\text { White et al. (2003); Gungor e } \\
\text { Gupta (1999); Pigosso et al. } \\
\text { (2010); Duflou et al., (2008); } \\
\text { Masanet e Horvath (2004) }\end{array}$ & $\begin{array}{l}\text { Computadores; Geral; Geral; geral; } \\
\text { Câmeras Fotográficas; Plástico } \\
\text { Proveniente de Eletrônicos (Material) }\end{array}$ \\
\hline & $\begin{array}{l}\text { Custo de Recuperação } \\
\text { do Produto }\end{array}$ & $\begin{array}{c}\text { Yu et al. (2000); Ferrão, Ribeiro } \\
\text { e Silva ( 2008); Pigosso et } \\
\text { al. (2010); Xanthopoulos e } \\
\text { lakovou (2009); Masanet e } \\
\text { Horvath (2004) }\end{array}$ & $\begin{array}{c}\text { Eletrônicos; Pneus; Geral; Geral; } \\
\text { Geral; Aquecedores Elétricos; } \\
\text { Plástico Proveniente de Eletrônicos } \\
\text { (Material) }\end{array}$ \\
\hline $\begin{array}{l}\text { Incentivos e } \\
\text { Regulamentação }\end{array}$ & $\begin{array}{l}\text { Incentivos } \\
\text { Governamentais para } \\
\text { Recuperação }\end{array}$ & $\begin{array}{l}\text { Ferrão, Ribeiro e Silva (2008); } \\
\text { Duflou et al. (2008); }\end{array}$ & $\begin{array}{l}\text { Pneus; Pneus; Pneus; Câmeras } \\
\text { Fotográficas; Câmeras Fotograficas }\end{array}$ \\
\hline
\end{tabular}

\section{AVALIAÇÃO DOS FCS POR ESPECIALISTAS}

Assim, estabelecido o número de quatro ou mais citações como o corte para obtenção dos FCS, foi possível obter um número de 10 fatores, sendo $25 \%$ (vinte e cinco por cento) de todos os FCS encontrados. Para melhor visualização destes fatores foi construído o quadro 4.
Para avaliar os FCS foi feita uma consulta a especialistas da área de desenvolvimento de produtos, que possuem conhecimento pleno sobre a fase final do ciclo de vida de produtos. A consulta teve como intuito a avaliação dos 10 FCS obtidos pela consulta bibliográfica, podendo ser excluí-

Quadro 4 - FCS mais Relevantes.

\begin{tabular}{|c|c|c|c|}
\hline Dimensão & $\begin{array}{c}\text { Fator Crítico de } \\
\text { Sucesso }\end{array}$ & Referência & Produtos \\
\hline \multirow{7}{*}{ Identificação } & $\begin{array}{c}\text { Identificação dos } \\
\text { Produtos (Marca, } \\
\text { Modelo) }\end{array}$ & $\begin{array}{c}\text { White et al. (2003); Goggin e } \\
\text { Browne, (1998); Johansson e } \\
\text { Brodin (2008); Oian e Zhang (2003) }\end{array}$ & $\begin{array}{c}\text { Computadores; Eletrônicos; } \\
\text { Elétricos e Eletrônicos; } \\
\text { Eletromecânicos }\end{array}$ \\
\cline { 2 - 4 } & $\begin{array}{c}\text { lakovou et al. (2009); lakovou } \\
\text { et al., (2009); Goggin e Browne } \\
\text { Identificação dos } \\
\text { Componentes }\end{array}$ & $\begin{array}{c}\text { (1998); Pigosso et al. (2010); Duval } \\
\text { e Maclean (2007); Grenchus et al. } \\
\text { (1998) }\end{array}$ & $\begin{array}{c}\text { Eletrônicos; Eletrônicos; Geral; } \\
\text { Plásticos Automotivos (Geral); } \\
\text { Geral }\end{array}$ \\
\cline { 2 - 4 } & Identificação dos & $\begin{array}{c}\text { Goggin e Browne (1998); Pigosso } \\
\text { et al., (2010); Johansson e Brodin } \\
\text { (2008); Grenchus et al. (1998) }\end{array}$ & $\begin{array}{c}\text { Eletrônicos; Geral; Elétricos e } \\
\text { Eletrônicos; Geral }\end{array}$ \\
\hline
\end{tabular}




\begin{tabular}{|c|c|c|c|}
\hline \multirow{4}{*}{$\begin{array}{l}\text { Eficiência na } \\
\text { Recuperação }\end{array}$} & $\begin{array}{l}\text { Peso de Material } \\
\text { Recuperado por } \\
\text { Produto }\end{array}$ & $\begin{array}{c}\text { lakovou et al. (2009); Wright, } \\
\text { Rahimifard e Clegg (2009); Pigosso } \\
\text { et al. (2010); Xanthopoulos e } \\
\text { lakovou (2009); Masanet e Horvath } \\
\text { (2004) }\end{array}$ & $\begin{array}{c}\text { Eletrônicos; Célula de } \\
\text { Combustível; Geral; Geral; Geral; } \\
\text { Aquecedores Elétricos; Plástico } \\
\text { Proveniente de Eletrônicos } \\
\text { (Material) }\end{array}$ \\
\hline & $\begin{array}{l}\text { Quantidade de Material } \\
\text { Recuperável por } \\
\text { Produto }\end{array}$ & $\begin{array}{c}\text { Yu et al. (2000); Ruhrberg } \\
\text { (2006); Ruhrberg (2006); Wright, } \\
\text { Rahimifard e Clegg (2009); White } \\
\text { et al. (2003); Pigosso et al. (2010); } \\
\text { Pigosso et al. (2010); Masanet e } \\
\text { Horvath (2004) }\end{array}$ & $\begin{array}{c}\text { Eletrônicos; Cobre (Material); } \\
\text { Cobre (Material); Células de } \\
\text { Combustível; Computadores; } \\
\text { Geral; Geral; Plástico } \\
\text { Proveniente de Eletrônicos } \\
\text { (Material) }\end{array}$ \\
\hline & $\begin{array}{l}\text { Tempo Necessário } \\
\text { para Recuperação do } \\
\text { Produto }\end{array}$ & $\begin{array}{c}\text { White et al. (2003); Gungor e } \\
\text { Gupta (1999); Pigosso et al. (2010); } \\
\text { Duflou et al., (2008); Masanet e } \\
\text { Horvath (2004) }\end{array}$ & $\begin{array}{l}\text { Computadores; Geral; Geral; } \\
\text { geral; Câmeras Fotográficas; } \\
\text { Plástico Proveniente de } \\
\text { Eletrônicos (Material) }\end{array}$ \\
\hline & $\begin{array}{c}\text { Custo de Recuperação } \\
\text { do Produto }\end{array}$ & $\begin{array}{l}\text { Yu et al. (2000); Ferrão, Ribeiro e } \\
\text { Silva ( 2008); Pigosso et al. (2010); } \\
\text { Xanthopoulos e lakovou (2009); } \\
\text { Masanet e Horvath (2004) }\end{array}$ & $\begin{array}{l}\text { Eletrônicos; Pneus; Geral; Geral; } \\
\text { Geral; Aquecedores Elétricos; } \\
\text { Plástico Proveniente de } \\
\text { Eletrônicos (Material) }\end{array}$ \\
\hline $\begin{array}{c}\text { Incentivos e } \\
\text { Regulamentação }\end{array}$ & $\begin{array}{l}\text { Incentivos } \\
\text { Governamentais para } \\
\text { Recuperação }\end{array}$ & $\begin{array}{l}\text { Ferrão, Ribeiro e Silva (2008); } \\
\text { Duflou et al. (2008); }\end{array}$ & $\begin{array}{l}\text { Pneus; Pneus; Pneus; Câmeras } \\
\text { Fotográficas; Câmeras } \\
\text { Fotograficas }\end{array}$ \\
\hline $\begin{array}{l}\text { Resíduos } \\
\text { Perigosos }\end{array}$ & $\begin{array}{c}\text { Presença de Resíduos } \\
\text { Perigosos }\end{array}$ & $\begin{array}{c}\text { Wright, Rahimifard e Clegg } \\
\text { (2009); Pigosso et al. (2010); } \\
\text { Xanthopoulos e lakovou (2009); } \\
\text { Duflou et al. (2008) }\end{array}$ & $\begin{array}{c}\text { Células de Combustível; Geral; } \\
\text { Aquecedores Elétricos; Câmeras } \\
\text { Fotográficas }\end{array}$ \\
\hline $\begin{array}{l}\text { Previsão de } \\
\text { Recuperação }\end{array}$ & $\begin{array}{c}\text { Previsão de } \\
\text { Recuperação Futura }\end{array}$ & $\begin{array}{l}\text { Ferrão, Ribeiro e Silva (2008); } \\
\text { Goggin e Browne (1998); Gungor } \\
\text { e Gupta (1999); Gehin, Zwolinski } \\
\text { e Brissaud, (2008); Masanet e } \\
\text { Horvath (2004) }\end{array}$ & $\begin{array}{l}\text { Pneus; Eletrônicos; Geral; } \\
\text { Eletrônicos e Elétricos; Plástico } \\
\text { Proveniente de Eletrônicos } \\
\text { (Material) }\end{array}$ \\
\hline
\end{tabular}

Fonte: Autores.

dos ou incluídos novos FCS pelos especialistas.

A avaliação foi feita por meio de um questionário, o qual foi composto por quatro questões: (1) questão relativa à comparação pareada entre os dez FCS analisados; (2) questão relativa à eliminação de algum FCS pelo respondente; (3) questão relativa à adição de algum FCS pelo respondente; (4) questão de opinião sobre os dez FCS analisados, sendo utilizada nesta a escala Likert.

Para validação dos FCS foi aplicado, no resultado da avaliação por escala Likert, o teste Kolmogorov-Smirnov (K-S). Segundo Mattar (1993), para um método estatístico não paramétrico, que utiliza escala de medição ordinal, e que possui disponibilidade das frequências relativas ou acumuladas (possível num teste com escala de Likert), cabe a aplicação do teste Kolmogorov-Smirnov (KS). O teste $\mathrm{K}-\mathrm{S}$ serve para indicar a rejeição de determinado item através de uma pesquisa com escala Likert.

Analisando ao todo o questionário aplicado foi possível obter algumas conclusões. Primeiramente é possível inferir que houve uma grande tendência de rejeição do FCS Previsão de Recuperação Futura. Três dos sete respondentes $(42,85 \%)$ indicaram a eliminação deste FCS. Além disso, por meio do teste K-S, foi possível assinalar a rejeição deste pelos respondentes. Seguindo a análise do questionário foi possível obter três possíveis FCS, os quais foram apresentados na questão 3, sendo estes: (1) Aplicabilidade ou reutilização do componente na montagem de produtos novos; (2) Disponibilidade a longo prazo de informações técnicas confiáveis sobre os produtos a serem recuperados (internet etc.); (3) Tipo, quantidade e variedade de elementos de fixação a serem removidos para a recuperação de componentes e ou re- 
síduos perigosos.

Quanto a esses possíveis FCS, podemos assinalar uma reafirmação da importância dos FCS 1, 2 e 3, referentes às informações dos produtos, componentes e materiais recuperados/reutilizados.

Assim, é possível concluir que todos os 10 FCS identificados na literatura possuem importância, podendo estes serem complementados por mais 3 FCS indicados pelos especialistas. Contudo o FCS Previsão de Recuperação Futura mostrou fragilidade e pode ser desconsiderado segundo os especialistas.

\section{CONSIDERAÇÕES FINAIS}

O conjunto de FCS obtidos nesta pesquisa pode ser utilizado como ferramenta para empresas que aplicam processos de recuperação e reuso de materiais e componentes provenientes de produtos, podendo também servir como uma base para o desenvolvimento de indicadores para o gerenciamento dos processos envolvidos no final do ciclo de vida de produtos

Como discussão da pesquisa é possível inferir a importância de alguns aspectos com relação ao final do ciclo de vida do produto. As informações provenientes dos produtos, com relação aos materiais, componentes e métodos de desmontagem do produto foram identificadas como de grande importância para a viabilidade na recuperação de produtos. A presença de resíduos perigosos e incentivos do governo também podem ser destacados como aspectos chave para a implementação de programas de recuperação de produtos.

A continuidade desta pesquisa está sendo dada pela ampliação da consulta a especialistas, buscando as opiniões de especialistas de ramos específicos, com o intuito de adaptar os FCS às necessidades de determinados setores. Outro ponto importante para o estudo é a apresentação do trabalho para empresas que desenvolvam processos relacionados à recuperação e reuso de materiais e componentes, fazendo com que estas possam aplicar ou mesmo aprimorar seus métodos de gestão do final do ciclo de vida do produto.

\section{REFERÊNCIAS}

ABU BAKAR, M.S.; RAHIMIFARD, S. Computer-aided recycling process planning for end-of-life electrical and electronic equipment. Journal of Engineering Manufacture, v.221, p.1369-1374, 2007.

AMELIA, L. et al. Initiating automotive component reuse in Malaysia. Journal of Cleaner Production, v.17, p.1572-1579, 2009.

ANDREOLA, F. et al. Recycling of EOL CRT Glass into Ceramic Glaze Formulations and Its Environmental Impact by LCA Approach. International Journal of Life Cycle Assessment, v.12, n.6, p.448-454, 2007.

BELLMANN, K.; KHARE, A. Economic issues in recycling end-of-life vehicles. Technovation, v.20, n.12, p.677-690, 2000.

BETTLEY, A.; BURNLEY, S. Towards Sustainable Operations Management Integrating Sustainability Management into Operations Management Strategies and Practices. Handbook of Performability Engineering, p.875-904, 2008.

BORGMAN, C.L.; FURNER, J. Scholarly Communication and Bibliometrics. In B. Cronin (Ed.), Annual Review of Information Science and Technology, v.36. Medford, NJ: Information Today, p.3-72. 2002.

CAGNO, E.; MAGALINI, F.; TRUCCO, P. Modelling and planning of Product Recovery Network: the case study of end-of-life refrigerators in Italy. International Journal of Environmental Technology and Management, v.8, n.4, p.385404, 2008. 
CAUDILL, R.J. et al. A Lifecycle Environmental Study of the Impact of E-commerce on Electronic Products. In: Proceedings of the 2000 IEEE International Symposium on Electronics and the Environment. São Francisco, Estados Unidos. 2000. p.298-303.

CAUDILL, R.J.; DICKINSON, D.A. Sustainability and end-of-life product management: a case study of electronics collection scenarios. In: International Symposium on Electronics and the Environment. Scottsdale, Estados Unidos. 2004. p. 132-137

DICKINSON, D.A.; CAUDILL, R.J. Sustainable product and material end-of-life management: an approach for evaluating alternatives. In: IEEE International Symposium on Electronics and the Environment. 2003. p. 1538.

DIODATO, V. Dictionary of Bibliometrics. Haworth Press: Binghamton, NY, 1994.

DUFLOU, J.R. et al. Efficiency and feasibility of product disassembly: A case-based study. CIRP Annals - Manufacturing Technology, v.57, p.583-600, 2008.

DUVAL, D.; MACLEAN H.L. The role of product information in automotive plastics recycling: a financial and life cycle assessment. Journal of Cleaner Production, v.15, p.11581168, 2007.

FERRÃO, P.; RIBEIRO, P.; SILVA, P. A management system for end-of-life tyres: a portuguese case study. Waste Management, v.28, n.3, p.604-614, 2008.

FORSLIND, K.H. Implementing extended producer responsibility: the case of Sweden's car scrapping scheme. Journal of Cleaner Production, v.13, n.6, p.619-629, 2005.

FTHENAKIS, V.M. End-of-life management and recycling of PV modules. Energy Policy, v.28, n.14, p.1051-1058, 2000.
GEHIN, A.; ZWOLINSKI, P.; BRISSAUD, D. A tool to implement sustainable end-of-life strategies in the product development phase. Journal of Cleaner Production, v.16, p.566$576,2008$.

GIL, A.C. Como elaborar projetos de pesquisa. 4.ed. São Paulo: Atlas, 2002.

GOGGIN, K.; BROWNE, J. Electronic products recovery-PAWS, a BRITE-EURAM project. Computer Industry, v.36, n.1-2, p.65-74, 1998.

GRENCHUS, E. et al. Linking demanufacturing operations with Product DFE initiatives. Proceedings of the 1998 IEEE International Symposium on Electronics and the Environment, p.270-274, 1998.

GRUJICIC, M. Total Life Cycle-Based Materials Selection for Polymer Metal Hybrid Body-in-White Automotive Components. Journal of Materials Engineering and Performance, v.18, n.2, p.111-128, 2009.

GUNGOR, A.; GUPTA, S.M. Issues in environmentally conscious manufacturing and product recovery: a survey. Computers \& Industrial Engineering, v.36, n.4, p.811-853, 1999.

HOEKSTRA, A. Y. The sustainability of a single activity, production process or product. Ecological Indicators, v.57, p. 82-84, 2015.

IAKOVOU, E. et al. A methodological framework for end-of-life management of electronic products. Resources, Conservation and Recycling, v.53, n.6, p.329-339, 2009.

IZARD, C.F.; MÜLLER, D.B. Tracking the devil's metal: Historical global and contemporary U.S. tin cycles. Resources, Conservation and Recycling, 2010.

JOHANSSON, G.; BRODIN, M.H. An analysis of product properties affecting performance of end-of-life systems for electrical and electronics equipment. Management of Envi- 
ronmental Quality: An International Journal, v.19, n.6, p.705-717, 2008.

KAHHAT, R. et al. Exploring e-waste management systems in the United States. Resources, Conservation and Recycling, v.52, n.7, p.955-964, 2008.

KOTLER, P. Administração de Marketing. São Paulo: Atlas, 1996.

KUMAR, S.; PUTNAM V. Cradle to cradle: reverse logistics strategies and opportunities across three industry sectors. International Journal of Production Economics, v.115, n.2, p.305-315, 2008.

KUO, T.C. Combination of case-based reasoning and analytical hierarchy process for providing intelligent decision support for product recycling strategies. Expert Systems with Applications, v.37, n.8, p.5558-5563, 2010a.

KUO, T.C. The construction of a collaborative-design platform to support waste electrical and electronic equipment recycling. RobotiCs and Computer-Integrated Manufacturing, v.26, n.1, p.100-108, 2010b.

LASZEWSKI, L.; CAREY, T. Integrating Environmental Product Design into Inkjet Printing Supplies. In: Proceedings of 2004 International IEEE Conference on the Asian Green Electronics. Singapore. 2004. p.86-91.

LOW, M.K.; WILLIAMS, D.J.; DIXON, C. Manufacturing products with end-of-life considerations: an economic assessment to the routes of revenue generation from mature products. IEEE Transactions on Components, Packaging, and Manufacturing Technology, v.21, n.1, p.4-10, 1998.

MANGUN, D.; THURSTON, D.L. Incorporating Component Reuse, Remanufacture, and Recycle Into Product Portfolio Design. IEEE Transactions on Engineering Management, v.49, n.4, p.479-490, 2002

MANZINI, E.; VEZZOLI, C. O desenvolvimento de produtos sustentáveis: os requisitos ambientais dos produtos industriais. São Paulo: Edusp, 2002.

MASANET, E.R.; HORVATH, A. A Decision-Support Tool for the Take-Back of Plastics from End-of-Life Electronics. In: Proceedings of the International Symposium on Electronics and the Environment. Washington, Estados Undidos. 2004. p.51-56

MATTAR, F.N. Pesquisa de Marketing. Volume 2. São Paulo: Atlas, 1993.

NAKAMURA, S.; KONDO, Y. A waste input-output life-cycle cost analysis of the recycling of end-of-life electrical home appliances. Ecological Economics, v.57, n.3, p.494-506, 2006.

OSIBANJO, O.; NNOROM, I.C. Material flows of mobile phones and accessories in Nigeria: environmental implications and sound end-of-life management options. Environmental Impact Assessment Review, v.28, n.2-3, p.198-213, 2008.

PAGELL, M.; WU, Z.; MURTHY, N.N. The supply chain implications of recycling. Business Horizons, v.50, n.2, p.133-143, 2007.

PARSONS, D. The Environmental Impact of Disposable Versus Re-Chargeable Batteries for Consumer Use. International Journal of Life Cycle Assessment, v.12, n.3, p.197-203, 2007.

PERALTA, G.L.; FONTANOS, P.M. E-waste issues and measures in the Philippines. Journal of Material Cycles and Waste Management, v.8, p.34-39, 2006.

PIGOSSO, D.C.A. et al. Ecodesign methods focused on remanufacturing. Journal of Cleaner Production, v.18, n.1, p.21-31, 2010. 
QIAN, X.; ZHANG, H.C. An environmentally conscious management model for end-of-life electromechanical products. In: Proceedings of the IEEE International Symposium on Electronics and the Environment. Washington, Estados Unidos. 2003. p.347-351.

RAHIMIFARD, S.; ABU BAKAR, M.S.; WILLIAMS, D.J. Recycling process planning for the End-of-Life management of waste from electrical and electronic equipment. CIRP Annals - Manufacturing Technology, v.58, n.1, p.5-8, 2009.

ROSE, C.M.; ISHII, K.; STEVELS, A. ELDA and EVCA: Tools for building product End-of-Life Strategy. The Journal of Sustainable Product Design, v.1, p.181-195, 2001.

RUHRBERG, M. Assessing the recycling efficiency of copper from end-of-life products in Western Europe. Resources, Conservation and Recycling, v.48, n.2, p.141-165, 2006.

SAKAI, S.; NOMA, Y.; KIDA, A. End-of-life vehicle recycling and automobile shredder residue management in Japan. Journal of Material Cycles and Waste Management, v.9, p.151-158, 2007.

SAKAI, S.; NOMA, Y.; KIDA, A. End-of-life vehicle recycling and automobile shredder residue management in Japan. Journal of Material Cycles and Waste Management, v.9, p.151-158, 2007.

SROGI, K. An overview of current processes for the thermochemical treatment of automobile shredder residue. Cleaner Technology Environmental Policy, v.10, p.235-244, 2008.

SUTHERLAND, J.W.; GUNTER, K.L.; WEINMANN, K.J. A model for improving economic performance of a demanufacturing system for reduced product end-of-life environmental impact. CIRP Annals - Manufacturing Technology, v.51, n.1, p.45-48, 2001.
UM, J.; YOON, J.; SUH, S. An architecture design with data model for product recovery management systems. Resources, Conservation and Recycling, v.52, n.10, p.1175-1184, 2008.

VANTI, N. Da bibliometria à webometria: uma exploração conceitual dos mecanismos utilizados para medir o registro da informação e a difusão do conhecimento. Ciência da Informação. Vol.31, n.2. 2002.

WHITE, C.D. et al. Product recovery with some byte: an overview of management challenges and environmental consequences in reverse manufacturing for the computer industry. Journal of Cleaner Production, v.11, n.4, p.445-458, 2003.

WRIGHT, E.I.; RAHIMIFARD, S.; CLEGG, A.J. Impacts of environmental product legislation on solid oxide fuel cells. Journal of Power Sources, v.190, n.2, p.362-371, 2009.

XANTHOPOULOS, A.; IAKOVOU, E. On the optimal design of the disassembly and recovery processes. Waste Management, v.29, n.5, p.1702-1711, 2009.

YU, J.; WILLIAMS, E.; JU, M. Analysis of material and energy consumption of mobile phones in China. Energy Policy, v.38, n.8, p.41354141, 2010.

YU, Y. et al. A Decision-Making Model for Materials Management of End-of-Life Electronic Products. Journal of Manufacturing Systems, v.19, n.2, p.94-107, 2000.

ZWOLINSKI, P.; LOPEZ-ONTIVEROS, M.A.; BRISSAUD, D. Integrated design of remanufacturable products based on product profiles. Journal of Cleaner Production, v.14, n.1516, p.1333-1345, 2006. 